\title{
Transferencia de tecnología: consideraciones y desafíos en escenarios de globalización
}

\author{
Velásquez, Luis Alexis *
}

\begin{abstract}
Resumen
Los procesos de transferencia de tecnología implican el aprovechamiento de las innovaciones de las personas, así como el reto de asimilar tecnologías existentes, añadir algún valor agregado y eventualmente generar variantes que puedan ser comercializadas. El objetivo central de este artículo, es conceptualizar y examinar estos factores, considerando el impacto que tienen los escenarios actuales de globalización en estos procesos. Además del apoyo documental de rigor, este estudio se basa en una metodología de corte cualitativa, sustentada en una dinámica de talleres y experiencias académicas con colegas y estudiantes de postgrado. Los resultados apuntan a que es impensable iniciar un proceso de asimilación o comercialización a terceros de una determinada tecnología, sin considerar las nuevas realidades impuestas por actuales mercados globales, apuntalados por rápidos y cambiantes saltos tecnológicos, que han iniciado transformaciones radicales en el mundo empresarial de hoy. Se pudo constatar que al desarrollar y transferir nuevas tecnologías, es necesario considerar nuevas realidades económicas, que ahora emergen bajo la óptica de escenarios cada vez más colaborativos y que requieren un nuevo orden de liderazgo global.
\end{abstract}

Palabras clave: Transferencia de Tecnología, Globalización, Tecnologías de Vanguardia.

Recibido: 08-04-10. Aceptado: 12-08-10

* $\quad$ PhD in Engineering, Magíster en Gerencia, Profesor Titular del Departamento de Ciencia y Tecnología, Universidad Nacional Experimental de Guayana (UNEG), Puerto Ordaz, Venezuela. E-mail: vluis@uneg.edu.ve; lavelz@hotmail.com; Telf.: 0414 - 8706185. 


\title{
Technology Transfer: Considerations and Challenges in Globalization Scenarios
}

\begin{abstract}
Technology transfer processes imply, first, taking advantage of people's innovations and second, becoming aware of current technologies, incorporating added value and, eventually, proposing potential alternatives that could be commercialized. This article examines these concepts, considering the impact of contemporary global scenarios on these processes. A qualitative methodology was applied. Further to the bibliographical and documental review, academic contributions coming from discussion groups involving postgraduate students and collaborators, mostly on technology transfer and the knowledge management domain, have been a significant support to this investigation. An analysis of the evolution and consequences of globalization on our cultures is carried out, identifying critical areas, focused mainly, though not exclusively, on the communication and information technology arena. As a result of this research, it was found that it is crucial to consider new economic realities coming from increasingly collaborative markets that require a new sort of global leadership in order to develop and transfer new technology.
\end{abstract}

Key words: Technology transfer, globalization, cutting-edge technologies.

\section{Introducción}

La tecnología, adecuadamente orientada, incide directamente en una mejor calidad de vida de los integrantes de una sociedad. Según Ohmae (2008), vivimos en un mundo cada vez más entrelazado e interdependiente, unidos por una economía global, lo cual ha sido posible en gran parte gracias a los adelantos tecnológicos, en particular los avances relacionados con el área de las Tecnologías de Información y Comunicaciones (TICs). Las organizaciones actuales empiezan a valorar cada vez más la importancia del capital tecnológico, es decir, de poseer plena confianza y entendimiento de la tecnología que adquieren, y en saber cómo aplicarla de manera óptima. Pero igualmente importante les resulta incorporar valor agregado a la tecnología asimilada a través de una combinación de experiencia, investigación e innova- ción y en su capacidad de transferir este valor agregado de manera oportuna, social y económicamente rentable. En otras palabras, el retorno sobre la inversión tecnológica, producto de transferir dicha tecnología puede transformarse en un factor clave de apalancamiento que impacta el crecimiento de éstas organizaciones. Aunque algunos autores (Allen, K., Goldberg, D. y Trout, J.) han realizado importantes contribuciones acerca de este complejo proceso de transferencia de tecnología, es escasa la literatura existente en relación a la asimilación o comercialización de nuevas tecnologías en escenarios impactados por una creciente globalización, donde el acceso a los mercados y la relación con proveedores, clientes y potenciales socios se ve ahora influenciada por estos ambientes cada vez más virtuales, altamente colaborativos y donde el conocimiento está dejando de ser un activo privado para 
Transferencia de tecnología: consideraciones y desafíos en escenarios de globalización Velásquez, Luis Alexis

convertirse en un activo compartido y de uso masivo a través de comunidades interconectadas globalmente. Actualmente es impensable iniciar un proceso de asimilación o comercialización a terceros de una determinada tecnología, sin considerar las nuevas realidades impuestas por estos mercados globales, a su vez apuntalados por rápidos y cambiantes saltos tecnológicos, que han iniciado transformaciones radicales en el mundo empresarial de hoy. En este artículo se analizan estas realidades, pasando por exponer conceptos fundamentales asociados a procesos de transferencia de tecnología, tanto internos como su comercialización a terceros, factores históricos que han influido en los actuales procesos de globalización, su contextualización en el presente y desafíos a considerar. Se le concede una alta importancia al segmento de productos y tecnologías de punta y su influencia como agentes globalizadores de la economía moderna. Esta investigación se fundamenta en una metodología descriptiva de tipo documental que ha sido complementada con experiencias obtenidas a través de talleres e intercambios académicos.

\section{Transferencia de Tecnología: Fundamentos}

El proceso de transferir tecnología en entornos organizacionales forma parte y constituye uno de los pilares fundamentales dentro de la estrategia global de Gestión Tecnológica. Uno de los conceptos que permite identificar las etapas mediante las cuales cualquier tecnología es asimilada por la empresa es el sugerido por Gaynor (1998), para el cual la gestión tecnológica consiste primeramente en identificar o constatar la utilidad de una determinada tecnología en bien de sus intereses, para luego adquirirla, adecuarla a sus necesidades o procesos productivos, lograr que apoye y mejore sus procesos operativos y finalmente determinar su momento o periodo de obsolescencia, que le permita descartarla completamente o sustituirla en aras de procurar otra que mejor satisfaga la realidad de la empresa en ese momento. En (Navarro et al, 2006) se presenta un resumen de estas cinco (5) etapas:

i) Percepción, en la cual la organización se percata o es consciente de la utilidad de una determinada tecnología emergente, capaz de responder a sus necesidades. ii) Adquisición, en la cual se procura la tecnología, fase que involucra además el estudio previo de factibilidad técnica y económica, así como cualquier otro mecanismo que permita establecer criterios de aprobación que avalen dicha adquisición. iii) Adaptación, de gran importancia pues usualmente la tecnología adquirida no es inicialmente aplicada de manera inmediata en los procesos operativos de la empresa, se requiere un periodo de ajuste o adecuación de la misma, que garanticen su asimilación y posterior utilización. iv) Avance, cuando la tecnología está ya arraigada en los diferentes procesos productivos de la empresa y le permite avanzar en su dominio y obtener el crecimiento esperado. v) Abandono, que permite determinar el momento en el cual la tecnología que está aplicándose, pueda ser oportunamente desincorporada.

La aplicación de la última etapa mencionada, centrada en identificar este periodo de obsolescencia, no resulta fá- 
cil, pues además de los factores propios de la tecnología utilizada, existen otros elementos a considerar, como la variación de los mercados, los cambios en el hábito o preferencia de los consumidores, políticas de estado y revisión de la misión y/o visión de la organización, entre otros.

La transferencia de tecnología, entendida como un proceso que implica la conversión, aplicación o adecuación de las invenciones o ideas innovadoras de las personas para la obtención de beneficios o la generación de usos prácticos (Speser, 2006), también supone la asimilación de tecnologías existentes dentro de una organización, generar algún tipo de valor agregado y eventualmente producir variantes que puedan ser comercializadas a otros mercados o empresas. De acuerdo a este concepto podemos afirmar que transferir tecnología es un proceso que se sitúa de maneras distintas en las etapas iii) y $v$ ) de las mencionadas en el párrafo anterior, como básicas en una estrategia de gestión tecnológica. Considerando el primer enfoque, es decir, establecer estrategias que permitan que la nueva tecnología adquirida pueda ser transferida a todas las unidades de la organización que la requieran, es una labor de adecuación, ajuste progresivo y validación de resultados en todos los niveles susceptibles de su aplicación. Pero más allá de sufrir las modificaciones necesarias para su inmediata aplicación en las unidades operativas, eventualmente a esta tecnología se le puede incorporar valor agregado producto de las innovaciones de los trabajadores o usuarios de la misma, lo cual generaría alternativas atractivas y de interés para otras organi- zaciones, deseosas de evaluar la adquisición e implementación de tecnologías emergentes.

Este enfoque innovador igualmente puede ser aplicado en la etapa de obsolescencia, cuando se detectan indicios de que la tecnología que ha estado utilizándose, justifica su pronta desincorporación, para lo cual la organización transfiere a otras empresas el know-how que ha acumulado durante su experiencia de uso con la tecnología, con un valor agregado añadido que usualmente es producto de investigación y pruebas en sus procesos internos.

Ahora bien, la actividad de comercializar tecnología, está primeramente orientada por los mercados, tal como se muestra en la Figura 1. Son las necesidades de un mercado las que disparan de manera natural la importancia que pueda tener en un determinado momento una oferta tecnológica. Adicional se cuenta con estrategias de mercadeo y demostraciones en el ambiente real de producción que se complementan y convergen en un acuerdo deseado entre las partes, lo cual culmina en la venta de la tecnología.

Según un estudio llevado a cabo por Lanerak et al. (2004), en más de trescientas empresas holandesas de variada actividad industrial, se concluyó que aunque apoyados en eficientes tácticas de lanzamiento de nuevos productos, la clave para comercializar nuevas tecnologías, es determinar las necesidades del mercado (market-oriented). Hay que reconocer, sin embargo, el importante papel que en la actualidad desempeñan los expertos de mercadeo, que en muchas ocasiones son capaces de señalar o determinar necesidades en una población 


\section{Figura 1 \\ Ley de la industria de Semiconductores de Moore}

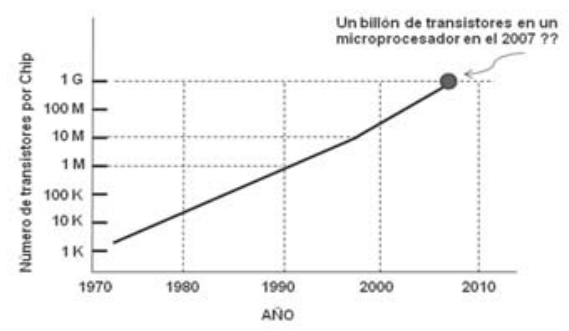

Fuente: Adaptado de Rafinejad (2007).

de consumidores o usuarios de servicios, que en un principio eran incapaces de reconocer tales necesidades. El extremo se presenta cuando estos expertos, siguiendo pautas netamente económicas, son capaces de sembrar estas necesidades, apoyándose en tácticas de persuasión, condicionamiento y exacerbación del ego de potenciales interesados (Diagrama 1).

Aunque en la Figura 1 se han mostrado los elementos primarios en un proceso de transferencia tecnológica, algunos autores han propuesto metodologías formales que involucran una gama mayor de consideraciones, en especial para la organización interesada en ofertar tecnología. Por ejemplo, Speser (2006) plantea un programa de doce pasos para lograr éxito al transferir tecnología de una organización a otra. Estos pasos se resumen a continuación:

1. Identificar los usuarios finales.

2. Entender sus necesidades, tanto las actuales, como aquellas nuevas que surgen o que cambian con el tiempo.

3. Determinar las fuerzas del mercado, que puedan influir de cualquier modo

\section{Diagrama 1 Elementos básicos de un proceso de Transferencia de Tecnología}

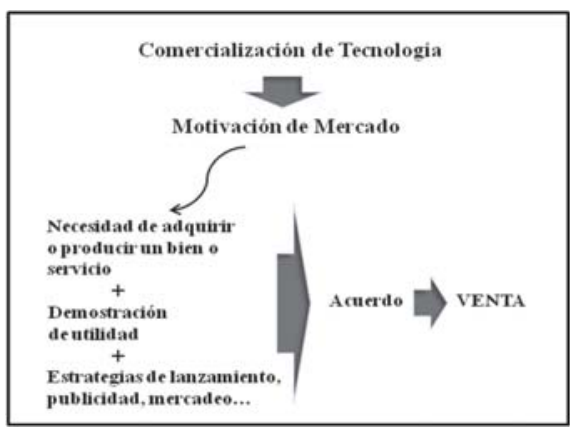

Fuente: Elaboración propia.

en la aceptación de la tecnología que se oferta.

4. Reconocer la competencia, al menos durante los cinco años posteriores a la entrada de la tecnología al mercado.

5. Identificar las barreras de entrada al mercado.

6. Determinar las ventajas competitivas relevantes. Si esto resulta complejo, tratar de ubicar otros usuarios finales en un ramo donde se esté consciente de que se tiene una ventaja competitiva diferenciada.

7. Ser honesto acerca de las fortalezas y debilidades que se poseen cuando se trata de poner en práctica las fases de desarrollo y comercialización.

8. Precisar las tácticas de lanzamiento que permitan lograr la venta a los usuarios finales.

9. Identificar las capacidades y los lapsos requeridos entre el momento de desarrollo de la tecnología y su lanzamiento o aprobación final. 
10. Encontrar otras organizaciones que puedan satisfacer las capacidades y lapsos mencionados en el paso anterior y que también desearían vender a los mismos usuarios finales, pero carecen de la tecnología apropiada.

11. Establecer alianzas con otros socios de manera que puedan impulsar la tecnología hacia mercados, en los que crear nuevos nichos les resulte más fácil.

12. Cerrar el acuerdo.

Como puede observarse, no todos los pasos considerados en el programa anterior deben cumplirse en su totalidad, por ejemplo puede que no sea atractivo establecer alianzas en un momento determinado, así como es posible que no existan otras organizaciones interesadas en vender el mismo tipo de tecnología. Tal como ocurre con otras metodologías o propuestas en esta área, las mismas constituyen solo pautas a seguir, un camino recomendado por especialistas que intentan considerar todas las variables que intervienen en este proceso usualmente complejo. Los principales aportes de esta metodología lo constituyen, primeramente, el hecho de considerar todas las variables y actores que intervienen, tales como identificación de necesidades, fortalezas y debilidades internas, usuarios finales, fuerzas del mercado, análisis de la competencia, lapsos de desarrollo/comercialización y posibilidad de alianzas. En segundo lugar, la metodología es genérica en el sentido que permite considerarse en empresas que operan en dominios muy diversos, en tercer lugar, no necesariamente requiere de una práctica secuencial de sus pasos, y, por último, su aplicación constituye en sí misma un ejercicio notable de colaboración e interrelación global tanto para el personal interno como entre sus potenciales clientes, competidores y socios.

Touhill et al. (2008), por el contrario, proponen una estrategia para comercializar tecnologías innovadoras, que inicia con el estímulo, la motivación y el desarrollo de ideas novedosas y luego se orienta al factor de desarrollo de la tecnología propiamente, desde su fase de diseño hasta su monitoreo y mejoras finales. La parte compleja de esta metodología y sobre la cual estos autores orientan gran parte de su estrategia, consiste en el reclutamiento y desarrollo de un personal altamente innovador donde estén presentes además de expertos en la tecnología, empresarios e inversionistas. Esto, aunque resulta ser una práctica muy razonable, especialmente en ambientes de desarrollo de tecnologías de punta que se caracterizan por cambios drásticos en el tiempo de vida útil de los productos ofertados, en la realidad no se traduce en una tarea sencilla de ejecutar por los encargados de reclutamiento y selección de personal. Otro aspecto crítico en el que los autores orientan su enfoque es hacia la identificación de áreas de interés para mercados potencialmente demandantes de los productos y tecnología generada en las mismas, para lo cual proponen la elaboración de un portafolio, donde se incluyan la mayor cantidad de ideas y ofertas atractivas que puedan ser comercializables. Esto igualmente requiere de una gran inversión en personal con visión vanguardista, en relación no sólo al conocimiento de actuales tecnologías de punta, sino también a las tendencias y rápidos avances que sobre la marcha se ori- 
Transferencia de tecnología: consideraciones y desafíos en escenarios de globalización Velásquez, Luis Alexis

ginan, así como de sólidas inversiones financieras. Otro elemento de gran importancia a considerar es el factor riesgo, el cual es usualmente considerado y controlado en función del capital disponible para invertir. Un indicador importante para considerar el nivel de riesgo a tomar en negociaciones tecnológicas, podría ser el retorno sobre la inversión que la empresa está dispuesta a aceptar como razonable en función del riesgo asumido.

\section{Globalización: realidades, evolución y efectos}

La globalización como fenómeno mundial significa que estamos en presencia de una reorganización de las sociedades, en las que las empresas e individuos no sólo compiten por un conocimiento global, sino que son ahora protagonistas de una disminución real en las barreras comerciales, culturales y sociales, que hasta hace apenas veinte años eran solo consideraciones teóricas. La globalización es un hecho que está cambiando de manera vertiginosa las estrategias de inversión de los países, la compra y gestión de nuevas tecnologías, la selección, formación y desarrollo del recurso humano $y$, en general, las actuales prácticas operativas empresariales (Pérez, 2006). Esta última década ha sido testigo de alianzas empresariales, fusiones y acuerdos de cooperación a nivel corporativo, que han impulsado el intercambio de productos y servicios en escenarios cada vez más globalizados, en los que algunos oficios dejan de ser útiles y otros emergen como necesarios, situaciones en que algunos trabajos o servicios son obligados a trasladarse a lugares remotos, ó donde la mano de obra es cambiada por otra más calificada y a un menor costo.

En su libro La Tierra es Plana, Friedman (2006) se refiere a este fenómeno como un aplanamiento del mundo, identificando tres grandes eras de la globalización a través de la historia, las cuales se muestran en el Cuadro 1.

En la primera era el autor destaca el rol de las naciones para participar y promover la competencia global, en la cual las oportunidades y la colaboración se establecían a través del impulso o apoyo de los países. En la segunda era el protagonismo en la integración global recae en las empresas multinacionales, a través de las cuales se logró una caída significativa en los costos de transporte, apoyados por el surgimiento y auge de la máquina de vapor y el ferrocarril, así como la caída en los costos de telecomunicaciones, gracias a la expansión del telégrafo, teléfonos, computadores personales, satélites, cables de fibra óptica y aparición de la primera versión de la World Wide Web. En la última y más reciente era, son los individuos a través de su conocimiento, los que tienen el poder y hacen posible a través de la colaboración con otros individuos, competir a escala global, es en este periodo donde se agudiza la subcontratación del conocimiento de estos individuos desde y hacia lugares remotos geográficamente y donde se evidencia un crecimiento tecnológico acelerado y el impacto mundial de potencias económicas como La India y China.

Los procesos de globalización han tenido adeptos y detractores a nivel mundial, con posiciones que los identifican con sistemas capitalistas y de corte neoliberal. Para Andrade (2007), convenir con 


\section{Tres (3) grandes Eras de la Globalización a lo largo de la historia}

\begin{tabular}{|c|c|c|}
\hline Periodo & Caracteristicas Resaltantes & Fuerza Impulsora \\
\hline $\begin{array}{c}\text { Era } 1 \\
1492-1800\end{array}$ & $\begin{array}{l}\text { Cristóbal Colón zarpa en busca de Las Indias. Se abre el } \\
\text { comercio entre el viejo y el nuevo mundo. } \\
\text { ¿ Cómo encaja mi país en la competencia y en las } \\
\text { oportunidades globales ? ¿ Cómo puedo globalizarme y } \\
\text { colaborar con otros a través de mi pais? }\end{array}$ & $\begin{array}{r}\text { PAISES en proceso } \\
\text { globalizador }\end{array}$ \\
\hline $\begin{array}{c}\text { Era } 2 \\
1800-2000\end{array}$ & $\begin{array}{l}\text { Integración global impulsada por: } \\
\text { Caída de costos en medios de Transporte y } \\
\text { Telecomunicaciones. } \\
\text { ¿Cómo encaja mi empresa en la economía globalizada? } \\
\text { ¿Cómo puedo globalizarme y colaborar con otros a través } \\
\text { demi empresa? }\end{array}$ & $\begin{array}{l}\text { EMPRESAS en } \\
\text { proceso globalizador }\end{array}$ \\
\hline $\begin{array}{r}\text { Era } 3 \\
>2000\end{array}$ & $\begin{array}{l}\text { Subcontratación del conocimiento de los individuos y } \\
\text { crecimiento acelerado de potencias económicas como } \\
\text { Chinaylalndia. } \\
\text { ¿ Cómo encajo yo en la competencia y en las } \\
\text { oportunidades de mi tiempo ? ¿ Cómo puedo yo solo } \\
\text { colaborar con otros individuosa escalaglobal? }\end{array}$ & $\begin{array}{l}\text { Poder de los } \\
\text { INDIVIDUOS para } \\
\text { competir y colaborar } \\
\text { a escala global. }\end{array}$ \\
\hline
\end{tabular}

Fuente: Elaboración propia con base en Friedman (2006).

procesos de globalización no necesariamente implica adoptar un pensamiento neoliberal, al igual que no toda práctica anti globalización es consecuencia de un análisis racional derivado de posiciones en contra de los procesos de expansión del neoliberalismo económico y financiero. El mismo autor sostiene que el predominio del neoliberalismo ha sido incentivado por los adelantos recientes en el área de tecnología de la información, los cuales a su vez han propiciado la justificación de elementos básicos que usualmente acompañan a los procesos de globalización, entre los que se encuentran la privatización, desregulación, offshoring, outsourcing, optimización de la cadena de suministros (supply-chaining) y el downsizing de organizaciones. Para este autor, estamos realmente en presencia de una globalización neoliberal que se expresa en políticas expansivas de producción capitalista.

Mato (2001), por otra parte, argumenta que tanto los detractores como los que respaldan la globalización, fetichizan eso que denominan globalización, ubicándola en una posición inaccesible que actúa con total independencia de los actores sociales, para lo cual es irrelevante si atribuyen su razón de ser a un azar del destino, o a fuerzas de carácter netamente económicas o tecnológicas. Lo cierto es que más allá del carácter neoliberal o no, la globalización nos ofrece la oportunidad para acceder a recursos geográficamente dispersos y poder para competir y colaborar a escala mundial, pero a la 
Transferencia de tecnología: consideraciones y desafíos en escenarios de globalización Velásquez, Luis Alexis

vez nos exige considerar una gama de nuevos factores y plantea serios retos que, de ser examinados y enfrentados oportunamente, les permitirá a las empresas ser exitosas en mercados con fronteras y barreras comerciales que la realidad nos muestra, son cada vez más inexistentes. Algunas de estas realidades que han permitido una disminución de las distancias en el plano económico, son magistralmente plasmadas por Friedman (2006), el cual afirma en sentido figurativo que ya la tierra no es redonda, debido particularmente a ciertas fuerzas que han actuado aplanando el mundo, entre las que destacan:

- La caída del muro de Berlín (1989).

- Netscape emerge como navegador en la Web (1995).

- Surgimiento de aplicaciones de informática para interconectar diversidad de operaciones y trabajos en la empresa (Workflow Software).

- Acceso libre a los códigos fuente (Open-Sourcing).

- Subcontratación para tareas especializadas (Out-Sourcing).

- Traslado de fábricas para abaratar costos (Offshoring).

- Cadena de suministros (Supply-Chaining).

- Incorporación de subcontratistas en actividades estratégicas de las empresas (Insourcing).

- Acceso libre a la información.

- Los Esteroides o adelantos en materia de tecnología inalámbrica y dispositivos de entrada/salida, procesamiento y almacenamiento de datos por computador.
Con respecto al muro de Berlín, su derrumbe permitió un aumento en el intercambio comercial y en general de conocimientos de diversa índole entre los países de Europa Oriental y el resto del mundo. Pero fue realmente la revolución informática representada por los adelantos en las TICs y en particular de los servicios de software, redes y protocolos de transmisión, los que permitieron a los individuos acceder e intercambiar información clave en su vida personal y de negocios. Aunque la compañía IBM introdujo el primer computador personal en 1981 y Microsoft lanzó al mercado su primera versión del sistema operativo Windows en 1985, es realmente el lanzamiento de Windows versión 3.0 en 1990 (solo unos pocos meses después de la caída del muro), lo que proporcionó a científicos y ciudadanos comunes equipados tan solo con un computador personal y un módem, la capacidad de interconectar los computadores con sus teléfonos y lograr entre otras cosas, enviar mensajes electrónicos vía Internet. Toda una revolución en el envío de información y comunicación utilizando esta plataforma, llegaría acompañada de nuevos servicios y a una tasa de crecimiento, inimaginable en aquel entonces. Igualmente, la $W e b$, propuesta por Tim-Berners-Lee en 1989, facilitó un ambiente de comunicación global, donde distancias geográficas, variedades culturales, idiomáticas y diferencias horarias ya no representaban un factor significativo a considerar en operaciones comerciales (Velásquez et al, 2003). En 1995, la empresa Netscape empieza a cotizar en la Bolsa con su producto Netscape Navigator, el cual sólo había permi- 
tido hasta ese entonces que algunos grupos de científicos e investigadores pudiesen compartir e intercambiar resultados de sus investigaciones. Rápidamente este navegador, al pasar al ámbito comercial, permitió el uso y la descarga masiva de archivos multimedia donde la accesibilidad a texto, imágenes, audio, animaciones y simulaciones ya eran posible a través de una plataforma vía Internet. También le dio un tremendo impulso al fenomenal despliegue y éxito que alcanzaron las denominadas empresas puntocom. La principal contribución del navegador Netscape en el aplanamiento mencionado se basaba principalmente en su capacidad para otorgarle una verdadera interoperabilidad a Internet. Luego saldría al mercado el sistema operativo Windows 95 con su navegador incorporado Internet Explorer, al igual que otros navegadores de otras empresas, para contribuir aún más a aumentar las opciones de navegabilidad de los usuarios a través de Internet. Hoy día existe una mayor disponibilidad de navegadores comerciales para acceder a la vasta red de información en Internet.

La aparición y consolidación de software informático que permitía a diferentes departamentos de una empresa, utilizando hardware de diferentes fabricantes, interconectar las diferentes actividades de la cadena de producción o ventas (Workflow Software), significó otro tremendo impulso a la globalización y surgimiento de empresas virtuales, donde las solicitudes de productos o servicios, o la realización de cada tarea podía ser realizada desde localizaciones remotas, por personas de diferente nacionalidad e incluso adaptándola a la conveniencia y horarios de los diferentes acto- res involucrados en el proceso de producción. Ya no era suficiente que una orden colocada en el departamento de ventas fuese automáticamente direccionada a otras instancias de la empresa como almacén o compras, facturación, selección de canales de distribución, envío del producto y la posterior medición de su nivel de satisfacción, utilizando software que interconectaba internamente estos departamentos, ahora cualquier segmento de esta cadena podía trasladarse a otro sitio, donde era atendido por personal subcontratado y especializado encargado de retornar soluciones que encajaban dentro de un proceso global. Friedman (2006) describe perfectamente esta situación mediante un ejemplo de la industria cinematográfica, en el cual un actor podía estar filmando una escena en un país $P$, atendiendo un libreto cuyo guión fue escrito y enviado desde una ciudad o país diferente que podríamos denominar $C$, acompañada de una animación computarizada llevada a cabo en una localización $X$ y la supervisión de sesiones interactivas de rodaje/escritura/animación desde otra ciudad o país distinto a los anteriores.

En relación al acceso abierto a códigos fuente o surgimiento de los denominados movimientos de software libre, desde las décadas de 1960s/1970s emergen respaldados por una comunidad de científicos y profesionales de la informática, que de manera colaborativa y sin fines de lucro desarrollan aplicaciones y programas que pueden ser descargados gratuitamente a través de Internet, permitiendo editar, modificar y añadir mejoras al código fuente, así como interactuar con la comunidad de desarrolladores pioneros de la aplicación y con los nuevos 
Transferencia de tecnología: consideraciones y desafíos en escenarios de globalización Velásquez, Luis Alexis

que se incorporan, posibilitando la actualización y mejora continua de la misma. Entre las aplicaciones de descarga gratuita y uso mundial más importantes desarrolladas en software libre, se tienen el programa para tecnología de servidores Web denominado Apache, el sistema operativo Linux, la enciclopedia online Wikipedia, el sistema de gestión de aprendizajes en el contexto de educación a distancia Moodle y el navegador Web Mozilla Firefox. Por una parte, el surgimiento de estas aplicaciones contribuyen a enfrentar los monopolios de grandes empresas con sus productos propietarios que exigen el pago de licencias por su uso, pero por otra, la continuidad en aspectos como la calidad, soporte técnico y actualización de nuevas versiones es cuestionada por estas empresas privadas, que sostienen que a la larga, sin los ingresos provenientes de la venta de estas aplicaciones, no sería posible realizar inversiones regulares en investigación e innovación, así como consideran incierta la sostenibilidad en el tiempo de esta comunidad voluntaria de científicos y desarrolladores de soluciones de software. El hecho cierto es que el aporte de estas personas ha impactado la manera en que nuevas soluciones informáticas están ahora disponibles en el mercado mundial, así como ha estimulado el trabajo colaborativo de comunidades de personas muy distantes entre sí, permitiendo consolidar aún más el fenómeno de la globalización y las soluciones compartidas.

Otro agente importante impulsor del proceso de globalización ha sido la subcontratación de servicios y trabajos para solucionar necesidades particulares en la cadena de actividades de negocios de una empresa. La idea consiste en delegar algunas funciones específicas de una empresa para que sean llevadas a cabo por otras compañías y reinsertar estos resultados en el proceso integral del negocio. La característica distintiva es que estas empresas o individuos prestatarios del servicio, pueden estar ubicadas en un continente distinto a las empresas contratantes. Las razones para esta subcontratación van desde una reducción significativa en los tiempos de respuesta ante un requerimiento, disminución de costos de mano de obra y gastos de instalación y alojamiento, hasta la eventual carencia o escasez local de profesionales con notable experticia en dominios particulares y formados con altos estándares de calidad. Por el contrario, el offshoring se produce cuando una empresa al constatar que las condiciones para producir un determinado bien o servicio le son más convenientes en otro lugar que puede estar geográficamente distante de su sede, traslada una de sus fábricas completas a ese sitio, manteniendo esencialmente sus mismas líneas de producción. Estas condiciones favorables estarían representadas por acceso a fuentes de energía más baratas, menores costos de mano de obra, deducciones en pagos de impuestos y beneficios sociales, así como prescindir de posibles conflictos con representaciones sindicales.

En cuanto a las cadenas de suministros, las mismas implican una interrelación y sincronización entre los sistemas de planificación de la producción y ventas, entre empresas y sus proveedores. Requiere el desarrollo e implementación de sistemas informáticos que permitan el seguimiento a las ventas y existencias de 
los inventarios, información que es continuamente compartida con los proveedores. El ejemplo más sencillo de esta modalidad de colaboración horizontal, se presenta cuando un cliente toma un producto cualquiera del mostrador de una tienda y lo lleva al punto de pago, donde este producto es identificado por un lector electrónico de código de barras u otro dispositivo de detección similar, el cual transmite una orden o aviso inmediato al sistema de información del proveedor, que impacta de manera instantánea toda una cadena de manufactura y reposición de dicho producto. Este método de colaboración es muy útil para la empresa porque al disponer de sistemas que registren las compras realizadas por los clientes y los descuentos automáticos de los inventarios, se optimiza el proceso de reposición; a los proveedores esto les indica el momento oportuno para adaptar sus líneas de producción para atenuar o incrementar su producción en relación con el comportamiento de esta demanda dinámica. Una estrategia completa de funcionamiento eficiente de la cadena de suministros involucra optimizar además otros factores igualmente importantes, como la recepción de mercancía que puede provenir del exterior, activación de canales de distribución internos, logística de almacenamiento y colocación de los productos al alcance de los consumidores finales.

Otro factor que ha afianzado la cooperación y alianza global en el ámbito empresarial y comercial, ha sido la incorporación de compañías subcontratistas en actividades estratégicas del negocio de corporaciones o empresas trasnacionales. Esta situación se da cuando algunas empresas dotadas de eficientes y ágiles infraestructuras de servicios de índole diversa, absorben parte de las operaciones de las empresas contratantes y soportan funciones que conforman la logística de la cadena de suministros o de cara a la entrega de pedidos o constatación de la satisfacción del consumidor final. Esto permite a estas grandes corporaciones ya establecidas en el mercado, dedicarse a lo que mejor saben hacer dentro de su dominio, usualmente ocuparse de la manufactura de líneas de productos para un mercado específico, dejando las actividades colaterales a estos subcontratistas que de manera inevitable tienen que asimilar e involucrarse en todos los eslabones de la línea de producción de sus contratantes.

Sin lugar a dudas, uno de los factores más influyentes en el efecto aplanador del mundo moderno, ha sido el impresionante desarrollo de programas de apoyo al acceso libre a la información alrededor de todo el mundo. Sistemas de búsqueda como Google o Yahoo, desde hace años han dejado de ser sólo motores de búsqueda, para convertirse en completos sistemas de apoyo a millones de usuarios en docenas de idiomas, en una inmensa gama de tareas y requerimientos de información. Han permitido la creación de verdaderas comunidades de personas que utilizan estos websites para intercambiar conocimiento sobre temas de interés afines a los miembros de esa comunidad. Puede decirse que entrañan una verdadera revolución global, acercando a usuarios muy distantes geográficamente, en cuanto a facilitar el acceso y compartir información en temas de interés muy diverso como salud, religión, relaciones interpersonales, compras, re- 
Transferencia de tecnología: consideraciones y desafíos en escenarios de globalización Velásquez, Luis Alexis

creación, ciencia, política, economía y publicidad.

Finalmente, los llamados esteroides, son todo un conjunto de tecnologías que se han convertido en un factor significativo de apalancamiento e impulsor de todas las fuerzas mencionadas en esta sección. Estos adelantos, que aún hoy día son objeto de intensa investigación y desarrollo, están representados por las tecnologías inalámbricas, la miniaturización, digitalización e integración de multiplicidad de funciones en aparatos de comunicación cada vez más completos y portátiles, transmisión de voz vía protocolos de Internet (VolP), transmisión a través de fibra óptica, avances en la comunicación por videoconferencia, incremento en las capacidades de almacenamiento de información y sobre todo en la mejora a nivel de velocidad de procesamiento computacional. Sólo por mencionar algunos ejemplos, hemos pasado de valores en unidades de almacenamiento de información del orden de Kilobits $\left(10^{3}\right.$ bits) a Terabits $\left(10^{12}\right.$ bits), fibras ópticas permitiendo tasas de transferencia de terabits/seg, velocidades de microprocesadores que han mejorado desde la centésima de MIPS (millones de instrucciones por segundo) hasta superar los diez mil millones de MIPS y puertos USB que han logrado ampliar los estándares de interconectividad y descargas de información cada vez más rápidas.

\section{Producción y transferencia de tecnología de avanzada y su efecto globalizador}

La compleja actividad de producir o transferir productos altamente innovado- res o de aplicación de tecnologías de punta o avanzada (High-Tech), implica conocer muy bien las estrategias de desarrollo de nuevos productos, comercialización de tecnologías innovadoras y sobre todo disponer de un equipo multidisciplinario que incluya empresarios, expertos en tecnología, inversionistas y personal altamente motivado y dispuesto a interactuar en una atmósfera de constante creatividad e inventiva, todos ellos en condiciones de adaptarse a escenarios donde el período de vida útil de los productos ofertados es muy corta, en los que ahora la empresa, clientes y proveedores coexisten e interactúan en espacios virtuales de colaboración global, y, operando en mercados globalizados donde la tecnología disponible cambia a ritmos vertiginosos e incluso donde el competidor de hoy puede convertirse en el potencial aliado del mañana.

Rafinejad (2007) considera que el éxito en cualquier actividad de negocios en el siglo XXI, depende de la habilidad de la empresa para operar en mercados globales y ser a la vez eficiente en el ámbito local, del desarrollo y comercialización de productos ambiental o ecológicamente sostenibles y de una eficiente administración de sus recursos, todos estos factores apoyados por el enorme potencial de las TICs. Esto significa que el éxito de una empresa requiere una considerable integración de estrategias de negocios, mercados, tecnología y ubicación de recursos (particularmente financieros y de personal calificado), lo cual garantizaría una rentabilidad sostenida y un liderazgo global. Pero independientemente de la buena gerencia que actúe sobre estos factores, es fundamental obtener pro- 
ductos diferenciados y oportunos, esto significa que tengan la capacidad de solventar una necesidad que ningún otro producto de la competencia pueda satisfacer y además ser el primero en cubrirla.

La manufactura y transferencia de estos productos y tecnologías de avanzada, representan un segmento de mercado muy diferenciado, que ha propiciado cambios notables en el sector tecnológico, en la planificación de la producción de nuevos bienes/servicios y, en el mercadeo de los mismos. A nivel tecnológico la tasa de cambios experimentada por la industria de software, telecomunicaciones, bioingeniería, electrónica y mejora en los dispositivos móviles, ha sido impactante. La Figura 1 muestra el extraordinario incremento en el número de transistores por microprocesador, además de destacar la ley de Moore en la industria de semiconductores, la cual establece que la mejora computacional de los microprocesadores se duplica cada 18 meses.

En relación a la manufactura de nuevos productos, el ciclo de vida útil de los mismos ha disminuido dramáticamen- te, básicamente debido a su rápida obsolescencia tecnológica, lo cual se evidencia en la Figura 2, observándose de manera cronológica el progreso tecnológico alcanzado por algunas áreas o dominios, en relación con su ritmo cíclico de innovación. Cada una de estas etapas ha estado caracterizada primeramente por un incremento progresivo del arraigo de la tecnología, hasta llegar a un tope y luego declinar radicalmente debido a la influencia de la innovación tecnológica, en su mismo dominio, o en áreas afines.

El acortamiento significativo en la vida útil de productos después de ser lanzados al mercado de consumo, es predominante en áreas vinculadas con las TICs, como por ejemplo la evolución experimentada por los dispositivos de almacenamiento, pasando de los discos flexibles (floppy disks), a unidades zip (zip drives), discos compactos de lectura/escritura (CD/RW), DVDs y tecnología Bluray, hasta las tarjetas flash de memoria, todo ello en un período no mayor de 20 años. Lo mismo ha ocurrido en el área de entretenimiento donde sólo en el sector

Figura 2

\section{Actividades económicas y ciclos de reemplazo o innovación}

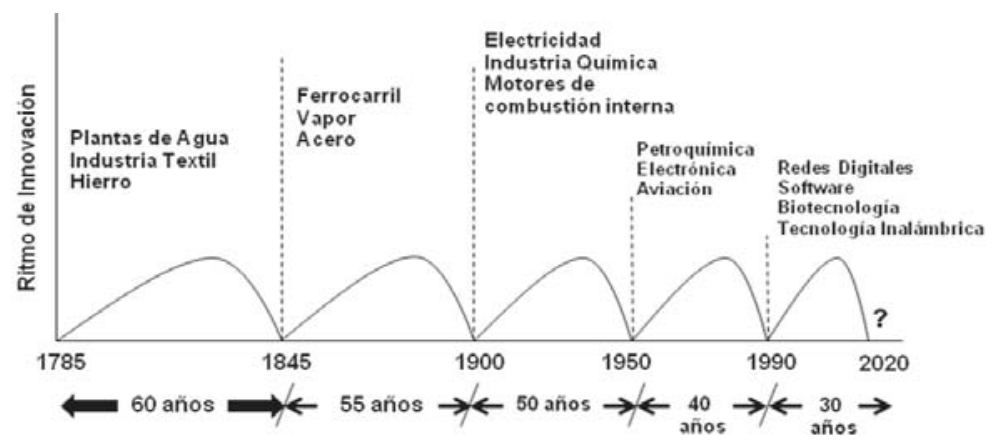

Fuente: Adaptado de Rafinejad (2007). 
Transferencia de tecnología: consideraciones y desafíos en escenarios de globalización Velásquez, Luis Alexis

de música hemos sido testigos de la transición de los formatos basados en acetato hasta los MP3/MP4.

Este notable progreso ha sido de tal magnitud que en muchas áreas no ha dado tiempo de crear y establecer estándares y en algunos casos los mismos no logran concretarse, al aparecer nuevas versiones de productos en tiempos muy cortos (Cooper, 2001). El acelerado ritmo de desarrollo tecnológico ha globalizado la industria de comunicaciones y transporte, creando nuevos servicios y productos industriales, así como la manera en que los bienes y servicios son mercadeados. Se ha reemplazado la manera tradicional en que algunas operaciones se llevan a cabo, ejemplos son la amplia gama de servicios bancarios vía Internet complementadas con las operaciones en los denominados cajeros automáticos (ATMs), tramitación de boletería electrónica en vuelos comerciales, compras variadas a través de tiendas virtuales como eBay y Amazon y oferta de novedosos productos de telefonía móvil, todo lo cual ha hecho posible que cada día más personas se integren a un gran mercado global y virtual.

\section{Conclusiones}

En esta investigación se ha evidenciado el profundo impacto que sobre actuales procesos de transferencia de tecnología, ejercen recientes escenarios de globalización, impulsados mayormente por los adelantos y un crecimiento vertiginoso de las TICs. Esto se ve reflejado en la manera en que nuevas prácticas operativas y estrategias, son ahora consideradas por empresarios, como la única op- ción para mantenerse a la vanguardia y competir en mercados económicos en los que ahora es requerido un nuevo tipo de liderazgo global, que implica examinar algunas variables y situaciones que antes no se reconocían como importantes y que a continuación se resumen.

Una de estas realidades, que está formando parte de muchas empresas en la actualidad, es la aplicación de estrategias orientadas a subdividir la cadena de producción, donde se generan tareas que son asignadas a quien esté en mejor capacidad de satisfacerlas, independientemente de su ubicación geográfica, lo cual significa que ahora el trabajo puede trasladarse a otra parte. Es el caso de individuos con una formación y el conocimiento necesarios en un dominio de interés particular, que están ahora en capacidad de ser subcontratados desde países diferentes al suyo y en una mejor posición de colaborar con otros individuos a escala global, impactando la realidad económica de estos países, de una manera impensable sólo décadas atrás. Esto se manifiesta a través de una subcontratación del conocimiento de estos individuos, donde algunas veces las diferencias geográficas constituyen una ventaja, pues las órdenes o pedidos demandados son requeridos en continentes con una diferencia horaria significativa con respecto al continente donde se satisface o realiza el servicio o trabajo, es decir, mientras el demandante duerme, el prestatario del servicio puede estar trabajando sobre la solicitud colocada el día anterior. Otra característica importante es la reducción significativa en los costos de alquileres y sueldos para este tipo de subcontrataciones, por ejemplo en países como La India o 
China, pueden llegar a ser significativamente menores que los de países europeos o de Norte América. Entre algunas de las áreas donde ya se destaca como una realidad la subcontratación de conocimiento se encuentran la Contabilidad, en el ramo de gestoría fiscal, a través de la elaboración de balances, declaraciones de renta y procesos varios de contabilidad general; en el sector Salud, con la realización de scanneres de apoyo al análisis de tomografías y resonancias magnéticas; en la parte de Periodismo, centrando el interés en cadenas de suministro de noticias, datos y estadísticas sobre ganancias empresariales y en información de última hora sobre oportunidades atractivas de negocios. Otro segmento importantísimo estimulado por el surgimiento de mercados globales y el rápido crecimiento en las TICs, es el de la industria de manufactura distribuida, el cual ha experimentado una profunda transformación. En el diseño, modelaje y simulación de partes de automóviles, aviones, viviendas y piezas de ensamble diversas, ahora intervienen equipos multidisciplinarios, de diferentes nacionalidades, que trabajan con modelaje geométrico de partes, desde sitios remotos y en estrecha colaboración, apoyados en plataformas con interfaces gráficas, multidimensionales y que permiten el manejo simultáneo de operaciones a distancia.

Todo este proceso actual de globalización del conocimiento, es y continuará siendo posible en gran medida debido a los significativos adelantos en materia de las TICs. Un ejemplo típico es la notable disminución en los costos de dispositivos móviles y en los tiempos de espera para accesos y descargas de información de interés, donde se ha avanzado desde la función básica de telefonía hasta la incorporación de una mayor gama de funciones que incluyen correo electrónico, navegación por Internet, manejo de agendas personales y de negocios, control del pago de servicios de condominio, operaciones bancarias y sesiones virtuales de comunicación con múltiples participantes y geográficamente dispersos, entre otras.

En economías donde las fronteras y barreras comerciales son cada vez más inexistentes, es igualmente fundamental para los nuevos gerentes alineados con este liderazgo global, comprender que el conocimiento es un activo clave cuyo impacto en las organizaciones depende en gran medida de la capacidad para conocer en un momento dado dónde se encuentra, quién lo posee y el medio para ubicarlo con prontitud, en ambientes de trabajo cada vez más colaborativos y eventualmente virtuales. Es precisamente en este punto, donde la influencia de Internet como proveedor mundial de grandes volúmenes de información, representa un ícono importante en la globalización de las sociedades actuales. El surgimiento de Internet y su adopción como un estándar internacional ha sido un factor impactante, al proveer una plataforma de comunicación global, donde distancias geográficas, idiomas y husos horarios, no constituyen factores críticos limitantes en actuales prácticas comerciales.

Otro factor importantísimo a tener en cuenta, lo constituye la proliferación y optimización de las cadenas de suministros, que han estimulado la colaboración a escala global, forzando a las empresas, fabricantes, subcontratistas y otros actores de la cadena de producción, a realizar 
Transferencia de tecnología: consideraciones y desafíos en escenarios de globalización Velásquez, Luis Alexis

una planificación de la producción integral y participativa y adoptar mecanismos estandarizados y consensuados por todos ellos, propiciando una interrelación más eficiente en sus eslabones productivos. Ello ha permitido, que al disminuir los costos de las empresas, se puedan abaratar los costos de los productos para el consumidor final o destinar más fondos a mejoras e innovación.

Como conclusión final, las nuevas realidades en materia de transferencia de tecnología apuntan, por una parte, a considerar la existencia y rápida expansión de mercados cada vez más globalizados y virtuales y, por otra, que las actividades económicas y el ciclo de vida útil de nuevos productos son profundamente impactados por el acelerado progreso tecnológico. Algunos campos donde este fenómeno es muy común están representados por avances en computación, medicina y biotecnología, los cuales están provocando el surgimiento o extensión de nuevas disciplinas y la promesa de nuevos horizontes, tal es el caso de la nanotecnología, el análisis del genoma humano y las mejoras en combustibles a base de hidrógeno. Más allá del provecho económico evidente que las iniciativas de transferencia de tecnología le otorgan a la organización, otros beneficios incluyen un incremento en la autonomía o independencia tecnológica, mayor proyección en el dinamismo de las actividades de investigación, mejoras en la formación y capacitación para los trabajadores, se amplían las fronteras en el conocimiento del área de negocios y se generan mayores posibilidades de diversificar los productos 0 servicios que se ofrecen, en otras palabras, coadyuvan al crecimiento integral de la organización.

\section{Referencias Bibliográficas}

Allen, Kathleen (2002). Bringing New Technology to Market, Prentice Hall, Upper Saddle River, New Jersey, USA.

Andrade, Jesús (2007). Sociedad de la información, lo público y lo privado de la información, Revista Venezolana de Gerencia, Año 12, No 39, Venezuela, Centro de Estudios de la Empresa, Universidad del Zulia, pp. 351-372.

Cooper, Robert (2001). Winning at New Products: Accelerating the Process from Idea to Launch, Editorial Perseus, Nueva York.

Friedman, Thomas (2006). La Tierra es Plana, breve historia del mundo globalizado del siglo XXI, Editorial Martínez Roca S.A., Madrid, España.

Gaynor, Gerard (1998). Manual de Gestión Tecnológica, Ediciones McGrawHill.

Goldberg, David (2006). The Entrepreneurial Engineer, Wiley \& Sons, NJ.

Langerak Fred, Hultink Erik y Robben Henry (2004). The Impact of Market Orientation, Product Advantage, and Launch Proficiency on New Product Performance and Organizational Performance, Journal of the Product Development Management Association, Vol. 21, No. 2, pp 84-98.

Mato, Daniel (2001). Des-fetichizar la "globalización”: basta de reduccionismos, apologías y demonizaciones, mostrar la complejidad y la práctica de los actores, Estudios latinoamericanos sobre cultura y transformaciones sociales en tiempos de globalización, UNESCO y Consejo Latinoamericano de Ciencias Sociales, Caracas, pp. 147-178.

Navarro Ketty, Romero Eunice, Bauza Roberto y Granadillo Víctor (2006). Estudio sobre la gestión tecnológica y del cono- 
cimiento en una organización creadora de conocimiento, Revista Venezolana de Gerencia, Año 11, No. 34, Venezuela, Centro de Estudios de la Empresa, Universidad del Zulia, pp. 262-276

Ohmae, Kenichi (2008). El Próximo Escenario Global, desafíos y oportunidades en un mundo sin fronteras, Editorial Norma S.A., Colombia.

Pérez, Carlota (2006). Hacia dónde va el mundo de hoy? Conocer la globalización desde la tecnología y la historia, Ediciones Urano, Caracas.
Rafinejad, Dariush (2007). Innovation, Product Development and Commercialization, J. Ross Publishing Inc., Fort Lauderdale, USA.

Speser, Phyllis (2006). The Art and Science of Technology Transfer, John Wiley \& Sons Inc, New Jersey, USA.

Touhill Joseph, Touhill Gregory y O'Riordan Thomas (2008). Commercialization of Innovative Technologies, John Wiley \& Sons Inc, New Jersey, USA.

Trout, Jack (2000). Differentiate or Die, John Wiley \& Sons Inc, New York. USA. 